\title{
Full characterization of graphs having certain normalized Laplacian eigenvalue of multiplicity $n-3$
}

\author{
Fenglei Tian*, Yiju Wang \\ School of Management, Qufu Normal University, Rizhao, China.
}

\begin{abstract}
Let $G$ be a connected simple graph of order $n$. Let $\rho_{1}(G) \geq \rho_{2}(G) \geq \cdots \geq$ $\rho_{n-1}(G)>\rho_{n}(G)=0$ be the eigenvalues of the normalized Laplacian matrix $\mathcal{L}(G)$ of $G$. Denote by $m\left(\rho_{i}\right)$ the multiplicity of the normalized Laplacian eigenvalue $\rho_{i}$. Let $\nu(G)$ be the independence number of $G$. In this paper, we give a full characterization of graphs with some normalized Laplacian eigenvalue of multiplicity $n-3$, which answers a remaining problem in [S. Sun, K.C. Das, On the multiplicities of normalized Laplacian eigenvalues of graphs, Linear Algebra Appl. 609 (2021) 365-385], i.e., there is no graph with $m\left(\rho_{1}\right)=n-3(n \geq 6)$ and $\nu(G)=2$. Moreover, we confirm that all the graphs with $m\left(\rho_{1}\right)=n-3$ are determined by their normalized Laplacian spectra.
\end{abstract}

Keywords: normalized Laplacian; normalized Laplacian eigenvalues; multiplicity of eigenvalues

AMS classification: $05 \mathrm{C} 50$

\section{Introduction}

Throughout, only connected and simple graphs are discussed. Let $G=(V(G), E(G))$ be a graph with vertex set $V(G)$ and edge set $E(G)$. Let $N_{G}(u)$ be the set of all the neighbors of the vertex $u$. Then $d_{u}=\left|N_{G}(u)\right|$ is called the degree of $u$. For a subset $S \subset V(G), S$ is called a set of twin points if $N_{G}(u)=N_{G}(v)$ for any $u, v \in S$. By $u \sim v$, we mean that $u$ and $v$ are adjacent. The distance of two vertices $u, v$ is denoted by $d(u, v)$ and the diameter of a graph $G$ is written as $\operatorname{diam}(G)$. A subset $S$ of $V(G)$ is called an independent set of $G$, if the vertices of $S$ induce an empty subgraph. The cardinality of the maximum independent set of $G$ is called the independence number, denoted by $\nu(G)$. The rank of a matrix $M$ is written as $r(M)$. Let $R_{v_{i}}$ be the row of $M$ indexed by the vertex $v_{i}$. The multiplicity of an eigenvalue $\lambda$ of $M$ is denoted by $m(\lambda)$. Denote by $\mathcal{G}(n, n-3)$ the set of all $n$-vertex $(n \geq 5)$ connected graphs with some normalized Laplacian eigenvalue of multiplicity $n-3$. Let $A(G)$ and $L(G)=D(G)-A(G)$ be the adjacency matrix and the Laplacian matrix of graph $G$,

\footnotetext{
${ }^{*}$ Corresponding author. E-mail address: tflqsd@qfnu.edu.cn. Supported by " the Natural Science Foundation of Shandong Province (No. ZR2019BA016)".
} 
respectively. Then the normalized Laplacian matrix $\mathcal{L}(G)=\left[l_{u v}\right]$ of graph $G$ is defined as

$$
\mathcal{L}(G)=D^{-1 / 2}(G) L(G) D^{-1 / 2}(G)=I-D^{-1 / 2}(G) A(G) D^{-1 / 2}(G),
$$

where

$$
l_{u v}= \begin{cases}1, & \text { if } u=v \\ -1 / \sqrt{d_{u} d_{v}}, & \text { if } u \sim v \\ 0, & \text { otherwise }\end{cases}
$$

For brevity, the normalized Laplacian eigenvalues are written as $\mathcal{L}$-eigenvalues. It is well known that the least $\mathcal{L}$-eigenvalue of a connected graph is 0 with multiplicity 1 (see [10]). Then let the $\mathcal{L}$-eigenvalues of a graph $G$ be

$$
\rho_{1}(G) \geq \rho_{2}(G) \geq \cdots \geq \rho_{n-1}(G)>\rho_{n}(G)=0
$$

The normalized Laplacian eigenvalues of graphs have been studied intensively (see for example $\left[1\left[\frac{8}{6}\right)\right.$, as it reveals not only some structural properties but also some relevant dynamical aspects (such as random walk) of graphs [10]. Recently, the multiplicity of the normalized Laplacian eigenvalues attracts much attention. Van Dam and Omidi [1] determined the graphs with some normalized Laplacian eigenvalue of multiplicity $n-1$ and $n-2$, respectively. Tian et al. [11,12] characterized some families of graphs of $\mathcal{G}(n, n-3)$, but the graphs with $\rho_{n-1}(G) \neq 1$ and $\nu(G)=\operatorname{diam}(G)=2$ that contain induced $P_{4}$ are not considered, which is the last remaining case. Sun and Das [9] presented the graphs of $\mathcal{G}(n, n-3)$ with $m\left(\rho_{n-1}(G)\right)=n-3$ and $m\left(\rho_{n-2}(G)\right)=n-3$ respectively, and gave the following problem.

Problem [9]: Is it true that there exists no connected graph with $m\left(\rho_{1}(G)\right)=n-3(n \geq 6)$ and $\nu(G)=2$ ?

To answer the above problem, it is urgent to complete the characterization of all the graphs in $\mathcal{G}(n, n-3)$. Note that the authors of [11,12] have obtained the following results.

Theorem 1.1. [11, 12] Let $G \in \mathcal{G}(n, n-3)$ be a graph of order $n \geq 5$. Then

(i) $\rho_{n-1}(G)=1$ if and only if $G$ is a complete tripartite graph $K_{a, b, c}$ or $K_{n}-e$, where $K_{n}-e$ is the graph obtained from the complete graph $K_{n}$ by removing an edge.

(ii) $\rho_{n-1}(G) \neq 1$ and $\nu(G) \neq 2$ if and only if $G \in\left\{G_{1}, G_{2}, G_{3}\right\}$ (see Fig. 1).

(iii) $\rho_{n-1}(G) \neq 1, \nu(G)=2$ and $\operatorname{diam}(G)=3$ if and only if $G=G_{4}$ (see Fig. 1).

(iv) $G$ is a cograph with $\rho_{n-1}(G) \neq 1$ and $\nu(G)=2$ if and only if $G=G_{5}$ (see Fig. 1).

Hence, to characterize all graphs of $\mathcal{G}(n, n-3)$ and to address the above problem in [9], it suffices to consider the graphs that contain induced path $P_{4}$ with $\rho_{n-1}(G) \neq 1$ and $\nu(G)=\operatorname{diam}(G)=2$. Here, we obtain the following conclusion.

Theorem 1.2. Let $G \in \mathcal{G}(n, n-3)$ be a graph of order $n \geq 5$. Then $G$ contains induced path $P_{4}$ with $\rho_{n-1}(G) \neq 1$ and $\nu(G)=\operatorname{diam}(G)=2$ if and only if $G$ is the cycle $C_{5}$. 
Remark 1.3. Combining Theorems 1.1 and 1.2, all graphs of $\mathcal{G}(n, n-3)$ are determined. As a result, the above problem in [9] is answered, that is, there is no connected graph with $m\left(\rho_{1}(G)\right)=n-3(n \geq 6)$ and $\nu(G)=2$. Now, we can also confirm the uncertain result in [9] that if $G$ is the graph with $m\left(\rho_{1}(G)\right)=n-3$ then $G$ is determined by its normalized Laplacian spectrum.

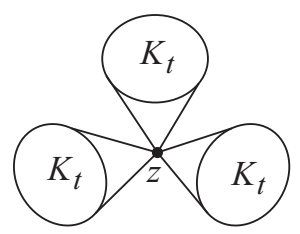

$G_{1}$

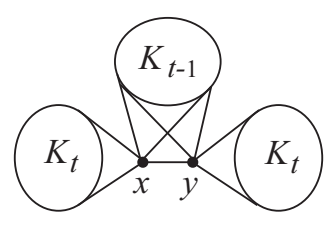

$G_{2}$

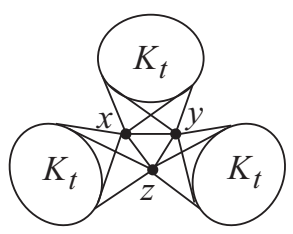

$G_{3}$

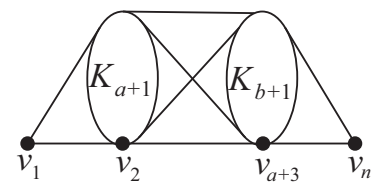

$G_{4}$

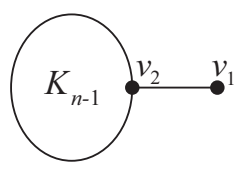

$G_{5}$

Fig. 1: The graphs $G_{i}(1 \leq i \leq 5)$.

The rest of the paper is arranged as follows. In Section 2, some lemmas are introduced. In Section 3, the proof of Theorem 1.2 is presented.

\section{Preliminaries}

For brevity, let $\Omega$ be the set of graphs of $\mathcal{G}(n, n-3)$ that contain induced path $P_{4}$ with $\rho_{n-1}(G) \neq 1$ and $\nu(G)=\operatorname{diam}(G)=2$. We always let $\theta$ be the $\mathcal{L}$-eigenvalue of $G$ with multiplicity $n-3$.

Lemma 2.1. [7] Let $G$ be a connected graph with order $n \geq 4$. If $G \not K_{p, q}, K_{a} \vee(n-a) K_{1}$ $(p+q=n, a \geq 2)$, then $\rho_{2}(G) \geq \frac{n-1}{n-2}$.

Lemma 2.2. 2] Let $G ¥ K_{n}$ be a connected graph with order $n \geq 2$. Then $\rho_{n-1}(G) \leq 1$.

Lemma 2.3. 5] Let $G$ be a graph with $n$ vertices. Let $K=\left\{v_{1}, \ldots, v_{q}\right\}$ be a clique in $G$ such that $N_{G}\left(v_{i}\right)-K=N_{G}\left(v_{j}\right)-K(1 \leq i, j \leq q)$, then $1+\frac{1}{d_{v_{i}}}$ is an $\mathcal{L}$-eigenvalue of $G$ with multiplicity at least $q-1$.

Lemma 2.4. 11] Let $G \in \mathcal{G}(n, n-3)$ with $\rho_{n-1}(G) \neq 1$, then $\theta \neq 1$.

Lemma 2.5. [12] Let $G \in \Omega$ with an induced path $P_{4}=v_{1} v_{2} v_{3} v_{4}$. Then

$$
(1-\theta)^{4} d_{v_{1}} d_{v_{2}} d_{v_{3}} d_{v_{4}}-\left(d_{v_{1}} d_{v_{2}}+d_{v_{3}} d_{v_{4}}+d_{v_{1}} d_{v_{4}}\right)(1-\theta)^{2}+1=0 .
$$

Moreover, if there is a vertex $u_{1}$ (resp., $u_{2}$ ) such that $u_{1} v_{2} v_{3} v_{4}$ (resp., $v_{1} u_{2} v_{3} v_{4}$ ) is also an induced path, then $d_{v_{1}}=d_{u_{1}}$ (resp., $\left.d_{v_{2}}=d_{u_{2}}\right)$.

The following lemma is useful for us to complete the proof of Theorem 1.2.

Lemma 2.6. Let $G \in \Omega$ and $H_{i}(1 \leq i \leq 5)$ be the graphs as shown in Fig. 2. Then the following assertions hold.

(i) If $G$ contains $H_{1}$ as an induced subgraph, then $1-\theta=-\frac{1}{d_{v_{1}}}=-\frac{1}{d_{v_{5}}}$. 
(ii) If $G$ contains $H_{2}$ as an induced subgraph, then $1-\theta=-\frac{1}{d_{v_{2}}}=-\frac{1}{d_{v_{5}}}$.

(iii) If $G$ contains $\mathrm{H}_{3}$ as an induced subgraph, then

$$
(1-\theta)^{2} d_{v_{1}} d_{v_{2}}+(1-\theta) d_{v_{4}}-1=0 .
$$

(iv) If $G$ contains $\mathrm{H}_{4}$ as an induced subgraph, then

$$
\left\{\begin{array}{l}
(1-\theta)=-\frac{d_{v_{3}}+d_{v_{5}}}{d_{v_{3}} d_{v_{2}}}=-\frac{d_{v_{2}}+d_{v_{4}}}{d_{v_{4}} d_{v_{5}}} \\
(1-\theta)^{2} d_{v_{1}}\left(d_{v_{3}}+d_{v_{5}}\right)+(1-\theta) d_{v_{3}}-1=0 .
\end{array}\right.
$$

(v) If $G$ contains $H_{5}$ as an induced subgraph, then

$$
(1-\theta)=-\frac{d_{v_{2}}+2 d_{v_{4}}}{d_{v_{4}}\left(d_{v_{2}}+d_{v_{5}}\right)}=-\frac{d_{v_{3}}+2 d_{v_{1}}}{d_{v_{1}}\left(d_{v_{3}}+d_{v_{5}}\right)}
$$

(vi) [12] If $G$ contains $H_{6}$ as an induced subgraph, then $1-\theta=-\frac{1}{d_{v_{1}}}=-\frac{1}{d_{v_{4}}}$.
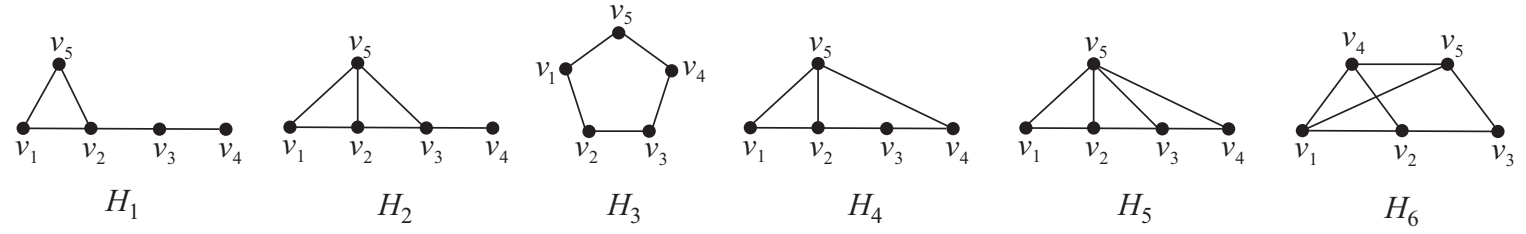

Fig. 2: The graphs $H_{i}(1 \leq i \leq 6)$.

Proof. We first show the proof of assertion (i).

Since $m(\theta)=n-3$, then $r(\mathcal{L}(G)-\theta I)=3$. Lemma 2.4 indicates that $\theta \neq 1$. Denote by $M_{1}$ the principal submatrix of $\mathcal{L}(G)-\theta I$ indexed by the vertices of $H_{1}$, then

$$
M_{1}=\left(\begin{array}{ccccc}
1-\theta & \frac{-1}{\sqrt{d_{v_{1}} d_{v_{2}}}} & 0 & 0 & \frac{-1}{\sqrt{d_{v_{1}} d_{v_{5}}}} \\
\frac{-1}{\sqrt{d_{v_{1}} d_{v_{2}}}} & 1-\theta & \frac{-1}{\sqrt{d_{v_{2}} d_{v_{3}}}} & 0 & \frac{-1}{\sqrt{d_{v_{2}} d_{v_{5}}}} \\
0 & \frac{-1}{\sqrt{d_{v_{2}} d_{v_{3}}}} & 1-\theta & \frac{-1}{\sqrt{d_{v_{3}} d_{v_{4}}}} & 0 \\
0 & 0 & \frac{-1}{\sqrt{d_{v_{3}} d_{v_{4}}}} & 1-\theta & 0 \\
\frac{-1}{\sqrt{d_{v_{1}} d_{v_{5}}}} & \frac{-1}{\sqrt{d_{v_{2}} d_{v_{5}}}} & 0 & 0 & 1-\theta
\end{array}\right) .
$$

One can easily obtain that the first three rows of $M_{1}$ are linearly independent (considering the minor indexed by the first three rows and the middle three columns of $M_{1}$ ), which yields that the rows $R_{v_{1}}, R_{v_{2}}, R_{v_{3}}$ of $\mathcal{L}(G)-\theta I$ are linearly independent, and then $R_{v_{5}}$ can be written as a linear combination of $R_{v_{1}}, R_{v_{2}}, R_{v_{3}}$. Let

$$
R_{v_{5}}=a R_{v_{1}}+b R_{v_{2}}+c R_{v_{3}}
$$


then

$$
\left\{\begin{array}{l}
a(1-\theta)-\frac{b}{\sqrt{d_{v_{1}} d_{v_{2}}}}=\frac{-1}{\sqrt{d_{v_{1}} d_{v_{5}}}} \\
\frac{-a}{\sqrt{d_{v_{1}} d_{v_{2}}}}+b(1-\theta)-\frac{c}{\sqrt{d_{v_{2}} d_{v_{3}}}}=\frac{-1}{\sqrt{d_{v_{2}} d_{v_{5}}}} \\
-\frac{b}{\sqrt{d_{v_{2}} d_{v_{3}}}}+c(1-\theta)=0 \\
-\frac{c}{\sqrt{d_{v_{3}} d_{v_{4}}}}=0
\end{array}\right.
$$

The fourth equation of (3) implies that $c=0$, and further $b=0$ from the third one. Recalling that $d_{v_{1}}=d_{v_{5}}$ by Lemma 2.5 , then we have $a=1$ from the second of (3), and thus $1-\theta=$ $-\frac{1}{d_{v_{1}}}=-\frac{1}{d_{v_{5}}}$ by the first of (프).

For assertion (ii), let

$$
M_{2}=\left(\begin{array}{ccccc}
1-\theta & \frac{-1}{\sqrt{d_{v_{1}} d_{v_{2}}}} & 0 & 0 & \frac{-1}{\sqrt{d_{v_{1}} d_{v_{5}}}} \\
\frac{-1}{\sqrt{d_{v_{1}} d_{v_{2}}}} & 1-\theta & \frac{-1}{\sqrt{d_{v_{2}} d_{v_{3}}}} & 0 & \frac{-1}{\sqrt{d_{v_{2}} d_{v_{5}}}} \\
0 & \frac{-1}{\sqrt{d_{v_{2}} d_{v_{3}}}} & 1-\theta & \frac{-1}{\sqrt{d_{v_{3}} d_{v_{4}}}} & \frac{-1}{\sqrt{d_{v_{3}} d_{v_{5}}}} \\
0 & 0 & \frac{-1}{\sqrt{d_{v_{3}} d_{v_{4}}}} & 1-\theta & 0 \\
\frac{-1}{\sqrt{d_{v_{1}} d_{v_{5}}}} & \frac{-1}{\sqrt{d_{v_{2}} d_{v_{5}}}} & \frac{-1}{\sqrt{d_{v_{3}} d_{v_{5}}}} & 0 & 1-\theta
\end{array}\right)
$$

be the principal submatrix of $\mathcal{L}(G)-\theta I$ indexed by the vertices of $H_{2}$. Similar as above discussion, one can assume that the Eq. (2) still holds. Then

$$
\left\{\begin{array}{l}
a(1-\theta)-\frac{b}{\sqrt{d_{v_{1}} d_{v_{2}}}}=\frac{-1}{\sqrt{d_{v_{1}} d_{v_{5}}}} \\
\frac{-a}{\sqrt{d_{v_{1}} d_{v_{2}}}}+b(1-\theta)-\frac{c}{\sqrt{d_{v_{2}} d_{v_{3}}}}=\frac{-1}{\sqrt{d_{v_{2}} d_{v_{5}}}} \\
-\frac{b}{\sqrt{d_{v_{2}} d_{v_{3}}}}+c(1-\theta)=\frac{-1}{\sqrt{d_{v_{3}} d_{v_{5}}}} \\
-\frac{c}{\sqrt{d_{v_{3}} d_{v_{4}}}}=0 .
\end{array}\right.
$$

By the fourth equation of (4), we see $c=0$. Further, recalling that $d_{v_{2}}=d_{v_{5}}$ for $H_{2}$ by Lemma 2.5, we get $b=1$ from the third one. Then $a=0$ from the first of (4), and thus $1-\theta=-\frac{1}{d_{v_{2}}}=-\frac{1}{d_{v_{5}}}$ by the second one.

For assertion (iii), let

$$
M_{3}=\left(\begin{array}{ccccc}
1-\theta & \frac{-1}{\sqrt{d_{v_{1}} d_{v_{2}}}} & 0 & 0 & \frac{-1}{\sqrt{d_{v_{1}} d_{v_{5}}}} \\
\frac{-1}{\sqrt{d_{v_{1}} d_{v_{2}}}} & 1-\theta & \frac{-1}{\sqrt{d_{v_{2}} d_{v_{3}}}} & 0 & 0 \\
0 & \frac{-1}{\sqrt{d_{v_{2}} d_{v_{3}}}} & 1-\theta & \frac{-1}{\sqrt{d_{v_{3}} d_{v_{4}}}} & 0 \\
0 & 0 & \frac{-1}{\sqrt{d_{v_{3}} d_{v_{4}}}} & 1-\theta & \frac{-1}{\sqrt{d_{v_{4}} d_{v_{5}}}} \\
\frac{-1}{\sqrt{d_{v_{1}} d_{v_{5}}}} & 0 & 0 & \frac{-1}{\sqrt{d_{v_{4}} d_{v_{5}}}} & 1-\theta
\end{array}\right)
$$

be the principal submatrix of $\mathcal{L}(G)-\theta I$ indexed by the vertices of $H_{3}$. Clearly, the middle three rows of $M_{3}$ are linearly independent, which yields that the rows $R_{v_{2}}, R_{v_{3}}, R_{v_{4}}$ of $\mathcal{L}(G)-\theta I$ 
are linearly independent, and then we set

$$
R_{v_{1}}=a R_{v_{2}}+b R_{v_{3}}+c R_{v_{4}}
$$

Applying (5) to the columns of $M_{3}$, we get

$$
\left\{\begin{array}{l}
\frac{-a}{\sqrt{d_{v_{1}} d_{v_{5}}}}=1-\theta \\
a(1-\theta)-\frac{b}{\sqrt{d_{v_{2}} d_{v_{3}}}}=\frac{-1}{\sqrt{d_{v_{1}} d_{v_{2}}}} \\
-\frac{b}{\sqrt{d_{v_{3}} d_{v_{4}}}}+c(1-\theta)=0 \\
-\frac{c}{\sqrt{d_{v_{4}} d_{v_{5}}}}=\frac{-1}{\sqrt{d_{v_{1}} d_{v_{5}}}}
\end{array}\right.
$$

The first and the fourth equations of (6) tell us that $a=-(1-\theta) \sqrt{d_{v_{1}} d_{v_{2}}}$ and $c=\sqrt{\frac{d_{v_{4}}}{d_{v_{1}}}}$, and further $b=(1-\theta) d_{v_{4}} \sqrt{\frac{d_{v_{3}}}{d_{v_{1}}}}$ from the third one. Taking the values of $a, b, c$ into the second of (6), we derive that $(1-\theta)^{2} d_{v_{1}} d_{v_{2}}+(1-\theta) d_{v_{4}}-1=0$, as required.

For assertion (iv), let

$$
M_{4}=\left(\begin{array}{ccccc}
1-\theta & \frac{-1}{\sqrt{d_{v_{1}} d_{v_{2}}}} & 0 & 0 & \frac{-1}{\sqrt{d_{v_{1}} d_{v_{5}}}} \\
\frac{-1}{\sqrt{d_{v_{1}} d_{v_{2}}}} & 1-\theta & \frac{-1}{\sqrt{d_{v_{2}} d_{v_{3}}}} & 0 & \frac{-1}{\sqrt{d_{v_{2}} d_{v_{5}}}} \\
0 & \frac{-1}{\sqrt{d_{v_{2}} d_{v_{3}}}} & 1-\theta & \frac{-1}{\sqrt{d_{v_{3}} d_{v_{4}}}} & 0 \\
0 & 0 & \frac{-1}{\sqrt{d_{v_{3}} d_{v_{4}}}} & 1-\theta & \frac{-1}{\sqrt{d_{v_{4}} d_{v_{5}}}} \\
\frac{-1}{\sqrt{d_{v_{1}} d_{v_{5}}}} & \frac{-1}{\sqrt{d_{v_{2}} d_{v_{5}}}} & 0 & \frac{-1}{\sqrt{d_{v_{4}} d_{v_{5}}}} & 1-\theta
\end{array}\right)
$$

be the principal submatrix of $\mathcal{L}(G)-\theta I$ indexed by the vertices of $H_{4}$. It is clear that the first three rows of $M_{4}$ are linearly independent, which indicates that the rows $R_{v_{1}}, R_{v_{2}}, R_{v_{3}}$ of $\mathcal{L}(G)-\theta I$ are linearly independent. Let

$$
R_{v_{5}}=a R_{v_{1}}+b R_{v_{2}}+c R_{v_{3}}
$$

Applying (7) to the columns of $M_{4}$, we have

$$
\left\{\begin{array}{l}
a(1-\theta)-\frac{b}{\sqrt{d_{v_{1}} d_{v_{2}}}}=\frac{-1}{\sqrt{d_{v_{1}} d_{v_{5}}}} \\
\frac{-a}{\sqrt{d_{v_{1}} d_{v_{2}}}}+b(1-\theta)-\frac{c}{\sqrt{d_{v_{2}} d_{v_{3}}}}=\frac{-1}{\sqrt{d_{v_{2}} d_{v_{5}}}} \\
\frac{-b}{\sqrt{d_{v_{2}} d_{v_{3}}}}+c(1-\theta)=0 \\
\frac{-c}{\sqrt{d_{v_{3}} d_{v_{4}}}}=\frac{-1}{\sqrt{d_{v_{4}} d_{v_{5}}}} \\
\frac{-a}{\sqrt{d_{v_{1}} d_{v_{5}}}}-\frac{b}{\sqrt{d_{v_{2}} d_{v_{5}}}}=1-\theta .
\end{array}\right.
$$


Combining the last three equations of (8), it follows that

$$
a=-\sqrt{d_{v_{1}} d_{v_{5}}}(1-\theta)\left(\frac{d_{v_{3}}}{d_{v_{5}}}+1\right), \quad b=d_{v_{3}}(1-\theta) \sqrt{\frac{d_{v_{2}}}{d_{v_{5}}}}, \quad c=\sqrt{\frac{d_{v_{3}}}{d_{v_{5}}}} .
$$

Taking the values of $a, b, c$ into the first and second equations of (8) respectively, one can easily derive that

$$
\left\{\begin{array}{l}
(1-\theta)^{2} d_{v_{1}}\left(d_{v_{3}}+d_{v_{5}}\right)+(1-\theta) d_{v_{3}}-1=0 \\
(1-\theta)=-\frac{d_{v_{3}}+d_{v_{5}}}{d_{v_{3}} d_{v_{2}}} .
\end{array}\right.
$$

Moreover, by the symmetry between $v_{2}$ and $v_{5}$ (resp., $v_{3}$ and $v_{4}$ ) in $H_{4}$, we can also get

$$
(1-\theta)=-\frac{d_{v_{4}}+d_{v_{2}}}{d_{v_{4}} d_{v_{5}}}
$$

At last, we prove assertion (v). Let the principal submatrix of $\mathcal{L}(G)-\theta I$ indexed by the vertices of $H_{5}$ be $M_{5}$, then

$$
M_{5}=\left(\begin{array}{ccccc}
1-\theta & \frac{-1}{\sqrt{d_{v_{1}} d_{v_{2}}}} & 0 & 0 & \frac{-1}{\sqrt{d_{v_{1}} d_{v_{5}}}} \\
\frac{-1}{\sqrt{d_{v_{1}} d_{v_{2}}}} & 1-\theta & \frac{-1}{\sqrt{d_{v_{2}} d_{v_{3}}}} & 0 & \frac{-1}{\sqrt{d_{v_{2}} d_{v_{5}}}} \\
0 & \frac{-1}{\sqrt{d_{v_{2}} d_{v_{3}}}} & 1-\theta & \frac{-1}{\sqrt{d_{v_{3}} d_{v_{4}}}} & \frac{-1}{\sqrt{d_{v_{3}} d_{v_{5}}}} \\
0 & 0 & \frac{-1}{\sqrt{d_{v_{3}} d_{v_{4}}}} & 1-\theta & \frac{-1}{\sqrt{d_{v_{4}} d_{v_{5}}}} \\
\frac{-1}{\sqrt{d_{v_{1}} d_{v_{5}}}} & \frac{-1}{\sqrt{d_{v_{2}} d_{v_{5}}}} & \frac{-1}{\sqrt{d_{v_{3}} d_{v_{5}}}} & \frac{-1}{\sqrt{d_{v_{4}} d_{v_{5}}}} & 1-\theta
\end{array}\right) .
$$

Similar as the discussion in assertion (iv), the Eq. (7) can still hold. Then applying (7) to the columns of $M_{5}$, we obtain

$$
\left\{\begin{array}{l}
a(1-\theta)-\frac{b}{\sqrt{d_{v_{1}} d_{v_{2}}}}=\frac{-1}{\sqrt{d_{v_{1}} d_{v_{5}}}} \\
\frac{-b}{\sqrt{d_{v_{2}} d_{v_{3}}}}+c(1-\theta)=\frac{-1}{\sqrt{d_{v_{3}} d_{v_{5}}}} \\
\frac{-c}{\sqrt{d_{v_{3}} d_{v_{4}}}}=\frac{-1}{\sqrt{d_{v_{4}} d_{v_{5}}}} \\
\frac{-a}{\sqrt{d_{v_{1}} d_{v_{5}}}}-\frac{b}{\sqrt{d_{v_{2}} d_{v_{5}}}}-\frac{c}{\sqrt{d_{v_{3}} d_{v_{5}}}}=1-\theta
\end{array}\right.
$$

It follows from the first three equations of (9) that

$$
a=\frac{d_{v_{3}}}{\sqrt{d_{v_{1}} d_{v_{5}}}}, \quad b=\sqrt{d_{v_{2}} d_{v_{3}}}\left((1-\theta) \sqrt{\frac{d_{v_{3}}}{d_{v_{5}}}}+\frac{1}{\sqrt{d_{v_{3}} d_{v_{5}}}}\right), \quad c=\sqrt{\frac{d_{v_{3}}}{d_{v_{5}}}} .
$$

Taking the values of $a, b, c$ into the last of (9), one can derive

$$
(1-\theta)=-\frac{d_{v_{3}}+2 d_{v_{1}}}{d_{v_{1}}\left(d_{v_{3}}+d_{v_{5}}\right)}
$$


Furthermore, the symmetry of $H_{5}$ implies that

$$
(1-\theta)=-\frac{d_{v_{2}}+2 d_{v_{4}}}{d_{v_{4}}\left(d_{v_{2}}+d_{v_{5}}\right)}
$$

as required.

For assertion (vi), one can refer to the process of proving Claim 1 of Lemma 3.2 in [12].

\section{Proof of Theorem 1.2}

Let $G \in \Omega$, i.e., $G$ is a graph of $\mathcal{G}(n, n-3)$ containing induced path $P_{4}$ with $\rho_{n-1}(G) \neq 1$ and $\nu(G)=\operatorname{diam}(G)=2$. Suppose $m(\theta)=n-3$ in $G$. Now we prove Theorem 1.2.

Proof of Theorem 1.2 By direct calculation, the normalized Laplacian spectrum of the cycle $C_{5}$ is $\left\{0.691^{2}, 1.809^{2}, 0\right\}$, then it follows that $G \in \Omega$. Thus the sufficiency is clear.

In the following, we present the necessity part. Suppose that $G \in \Omega$ and $m(\theta)=n-3$ in $G$, then $\theta \neq 1$ from Lemma 2.4. Denote by $P_{4}=v_{1} v_{2} v_{3} v_{4}$ an induced path of $G$. Assume that $U \subseteq V\left(P_{4}\right)$ and

$$
S_{U}=\left\{u \in V(G) \backslash V\left(P_{4}\right): N_{G}(u) \cap V\left(P_{4}\right)=U\right\}
$$

It follows from $\nu(G)=2$ that any vertex out of $V\left(P_{4}\right)$ must be adjacent to at least two of $V\left(P_{4}\right)$ and $S_{\left\{v_{1}, v_{3}\right\}}=S_{\left\{v_{2}, v_{4}\right\}}=S_{\left\{v_{2}, v_{3}\right\}}=\emptyset$. Further, since $\operatorname{diam}(G)=2$, then $d\left(v_{1}, v_{4}\right)=2$, and thus there exists a vertex, say $v_{5}$, adjacent to $v_{1}$ and $v_{4}$. Note that $v_{5}$ maybe belong to $S_{\left\{v_{1}, v_{4}\right\}}, S_{\left\{v_{1}, v_{2}, v_{4}\right\}}, S_{\left\{v_{1}, v_{3}, v_{4}\right\}}$ or $S_{\left\{v_{1}, v_{2}, v_{3}, v_{4}\right\}}$. Accordingly, the remaining proof can be divided into the following cases.

Case 1. Suppose that $v_{5} \in S_{\left\{v_{1}, v_{4}\right\}}$, i.e., $S_{\left\{v_{1}, v_{4}\right\}} \neq \emptyset$.

We will complete the discussion of this case by the following claims.

Claim $1.1\left|S_{\left\{v_{1}, v_{4}\right\}}\right|=1$ and $S_{\left\{v_{1}, v_{2}\right\}}=S_{\left\{v_{3}, v_{4}\right\}}=\emptyset$.

Suppose that $\left|S_{\left\{v_{1}, v_{4}\right\}}\right| \geq 2$, then all the vertices of $S_{\left\{v_{1}, v_{4}\right\}}$ induce a clique (otherwise, $\nu(G) \geq 3$, a contradiction). Then $G$ contains an induced subgraph isomorphic to $X_{1}$ in Fig. 3. Similarly, one can obtain that if $S_{\left\{v_{1}, v_{2}\right\}} \neq \emptyset$ or $S_{\left\{v_{3}, v_{4}\right\}} \neq \emptyset, G$ also contains an induced subgraph isomorphic to $X_{1}$. Since $X_{1}$ contains $H_{1}$ as an induced subgraph, then by Lemma 2.6 (i)

$$
1-\theta=-\frac{1}{d_{v_{1}}}
$$

Moreover, $C_{5}$ (i.e., $\left.H_{3}\right)$ is an induced subgraph of $X_{1}$, then by Lemma 2.6 (iii)

$$
(1-\theta)^{2} d_{v_{1}} d_{v_{2}}+(1-\theta) d_{v_{4}}-1=0
$$

Combining (10) and (11), we get

$$
d_{v_{2}}=d_{v_{1}}+d_{v_{4}}
$$


It is not hard to see that

$$
\left\{\begin{array}{l}
d_{v_{2}}=\left|S_{\left\{v_{1}, v_{2}\right\}}\right|+\left|S_{\left\{v_{1}, v_{2}, v_{3}\right\}}\right|+\left|S_{\left\{v_{2}, v_{3}, v_{4}\right\}}\right|+\left|S_{\left\{v_{1}, v_{2}, v_{4}\right\}}\right|+\left|S_{\left\{v_{1}, v_{2}, v_{3}, v_{4}\right\}}\right|+2 \\
d_{v_{1}}=\left|S_{\left\{v_{1}, v_{2}\right\}}\right|+\left|S_{\left\{v_{1}, v_{4}\right\}}\right|+\left|S_{\left\{v_{1}, v_{2}, v_{3}\right\}}\right|+\left|S_{\left\{v_{1}, v_{2}, v_{4}\right\}}\right|+\left|S_{\left\{v_{1}, v_{3}, v_{4}\right\}}\right|+\left|S_{\left\{v_{1}, v_{2}, v_{3}, v_{4}\right\}}\right|+1 \\
d_{v_{4}}=\left|S_{\left\{v_{3}, v_{4}\right\}}\right|+\left|S_{\left\{v_{1}, v_{4}\right\}}\right|+\left|S_{\left\{v_{2}, v_{3}, v_{4}\right\}}\right|+\left|S_{\left\{v_{1}, v_{2}, v_{4}\right\}}\right|+\left|S_{\left\{v_{1}, v_{3}, v_{4}\right\}}\right|+\left|S_{\left\{v_{1}, v_{2}, v_{3}, v_{4}\right\}}\right|+1
\end{array}\right.
$$

It follows from (12) and (13) that $\left|S_{\left\{v_{1}, v_{4}\right\}}\right|=0$, a contradiction.

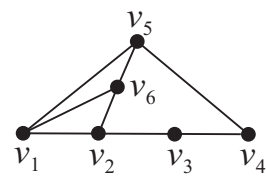

$X_{1}$

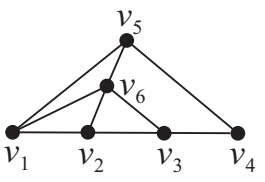

$X_{2}$

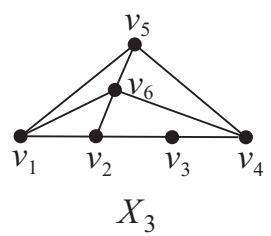

$X_{3}$

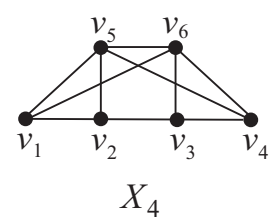

$X_{4}$

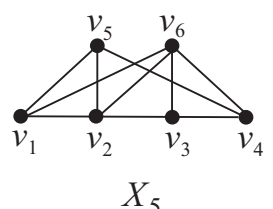

$X_{5}$

Fig. 3: The graphs $X_{i}(1 \leq i \leq 5)$.

Claim $1.2 S_{\left\{v_{1}, v_{2}, v_{3}\right\}}=S_{\left\{v_{2}, v_{3}, v_{4}\right\}}=\emptyset$.

It suffices to prove that $S_{\left\{v_{1}, v_{2}, v_{3}\right\}}=\emptyset$. Suppose for a contradiction that $S_{\left\{v_{1}, v_{2}, v_{3}\right\}} \neq \emptyset$ and $v_{6} \in S_{\left\{v_{1}, v_{2}, v_{3}\right\}}$. If $v_{5} \nsim v_{6}$, then the vertices $v_{i}(1 \leq i \leq 6)$ induce a subgraph isomorphic to $X_{1}$. One can obtain a contradiction by similar discussion as above. If $v_{5} \sim v_{6}$, then $X_{2}$ in Fig. 3 is an induced subgraph of $G$. Deleting $v_{3}$ with the incident edges from $X_{2}$, we also get (10) by Lemma 2.6 (ii). Analogous discussion as Claim 1.1, the Eq. (12) still holds. As $\left|S_{\left\{v_{1}, v_{4}\right\}}\right|=1$ and $S_{\left\{v_{1}, v_{2}\right\}}=S_{\left\{v_{3}, v_{4}\right\}}=\emptyset$ from Claim 1.1, then

$$
\left\{\begin{array}{l}
d_{v_{2}}=\left|S_{\left\{v_{1}, v_{2}, v_{3}\right\}}\right|+\left|S_{\left\{v_{2}, v_{3}, v_{4}\right\}}\right|+\left|S_{\left\{v_{1}, v_{2}, v_{4}\right\}}\right|+\left|S_{\left\{v_{1}, v_{2}, v_{3}, v_{4}\right\}}\right|+2 \\
d_{v_{1}}=\left|S_{\left\{v_{1}, v_{4}\right\}}\right|+\left|S_{\left\{v_{1}, v_{2}, v_{3}\right\}}\right|+\left|S_{\left\{v_{1}, v_{2}, v_{4}\right\}}\right|+\left|S_{\left\{v_{1}, v_{3}, v_{4}\right\}}\right|+\left|S_{\left\{v_{1}, v_{2}, v_{3}, v_{4}\right\}}\right|+1 \\
d_{v_{4}}=\left|S_{\left\{v_{1}, v_{4}\right\}}\right|+\left|S_{\left\{v_{2}, v_{3}, v_{4}\right\}}\right|+\left|S_{\left\{v_{1}, v_{2}, v_{4}\right\}}\right|+\left|S_{\left\{v_{1}, v_{3}, v_{4}\right\}}\right|+\left|S_{\left\{v_{1}, v_{2}, v_{3}, v_{4}\right\}}\right|+1
\end{array}\right.
$$

which, together with (12), yields that $\left|S_{\left\{v_{1}, v_{4}\right\}}\right|=0$, a contradiction.

Claim $1.3 S_{\left\{v_{1}, v_{2}, v_{4}\right\}}=S_{\left\{v_{1}, v_{3}, v_{4}\right\}}=\emptyset$.

We only need to show $S_{\left\{v_{1}, v_{2}, v_{4}\right\}}=\emptyset$. Assume that $S_{\left\{v_{1}, v_{2}, v_{4}\right\}} \neq \emptyset$ and $v_{6} \in S_{\left\{v_{1}, v_{2}, v_{4}\right\}}$, then $v_{5} \sim v_{6}$ (otherwise $\nu(G) \geq 3$, a contradiction). Thus $X_{3}$ in Fig. 3 is an induced subgraph of $G$. Removing $v_{4}$ with the incident edges from $X_{3}$, we get (10) again by Lemma 2.6 (ii). By analogous discussion as above, one can easily obtain a contradiction.

Claim $1.4 S_{\left\{v_{1}, v_{2}, v_{3}, v_{4}\right\}}=\emptyset$.

Combining the first three claims, we see that if $S_{\left\{v_{1}, v_{2}, v_{3}, v_{4}\right\}} \neq \emptyset$, then all the vertices of $V(G) \backslash\left\{v_{1}, \cdots, v_{5}\right\}$ belong to $S_{\left\{v_{1}, v_{2}, v_{3}, v_{4}\right\}}$. Then

$$
d_{v_{1}}=d_{v_{2}}=d_{v_{3}}=d_{v_{4}}
$$

Since $G$ now contains $H_{5}$ (see Fig. 2) as an induced subgraph, then by Lemma 2.6 (v)

$$
(1-\theta)=-\frac{d_{v_{2}}+2 d_{v_{4}}}{d_{v_{4}}\left(d_{v_{2}}+d_{v_{5}}\right)},
$$


which is a rational number. Furthermore, since $G$ contains an induced $C_{5}$, then by (11) and (14) we derive that

$$
(1-\theta)=\frac{-1 \pm \sqrt{5}}{2 d_{v_{1}}}
$$

which is an irrational number, a contradiction.

The above four claims indicate that if $S_{\left\{v_{1}, v_{4}\right\}} \neq \emptyset$, then $\left|S_{\left\{v_{1}, v_{4}\right\}}\right|=1$ and $|V(G)|=5$, i.e., $G$ is the cycle $C_{5}$.

Case 2. Suppose that $v_{5} \in S_{\left\{v_{1}, v_{2}, v_{4}\right\}}$, i.e., $S_{\left\{v_{1}, v_{2}, v_{4}\right\}} \neq \emptyset$ and $S_{\left\{v_{1}, v_{4}\right\}}=\emptyset$.

The following claims will help us complete the discussion of this case.

Claim 2.1 $S_{\left\{v_{1}, v_{2}\right\}}=S_{\left\{v_{3}, v_{4}\right\}}=\emptyset$.

We first demonstrate $S_{\left\{v_{1}, v_{2}\right\}}=\emptyset$. If $S_{\left\{v_{1}, v_{2}\right\}} \neq \emptyset$, say $v_{6} \in S_{\left\{v_{1}, v_{2}\right\}}$, then by Lemma 2.6 (i), the Eq. (10) holds. Since $G$ contains $H_{4}$ (see Fig. 2) as an induced subgraph, then by Lemma 2.6 (iv)

$$
\left\{\begin{array}{l}
(1-\theta)^{2} d_{v_{1}}\left(d_{v_{3}}+d_{v_{5}}\right)+(1-\theta) d_{v_{3}}-1=0 \\
(1-\theta)=-\frac{d_{v_{2}}+d_{v_{4}}}{d_{v_{4}} d_{v_{5}}}
\end{array}\right.
$$

By (10) and the first equation of (15), we get $d_{v_{1}}=d_{v_{5}}$, which implies that $(1-\theta)=-\frac{1}{d_{v_{5}}}$. Hence, by the second equation of (15), we have $d_{v_{2}}=0$, a contradiction.

Next, we prove that $S_{\left\{v_{3}, v_{4}\right\}}=\emptyset$. Suppose that $S_{\left\{v_{3}, v_{4}\right\}} \neq \emptyset$ and $v_{6} \in S_{\left\{v_{3}, v_{4}\right\}}$, then Lemma 2.6 (i) indicates that

$$
(1-\theta)=-\frac{1}{d_{v_{4}}}
$$

It follows from (16) and the second equation of (15) that $d_{v_{2}}+d_{v_{4}}=d_{v_{5}}$. Note that any vertex out of $V\left(P_{4}\right)$ must be adjacent to $v_{2}$ or $v_{4}$ (thanks to $\nu(G)=2$ ). Thus

$$
d_{v_{5}}=d_{v_{2}}+d_{v_{4}} \geq 3+3+n-6=n,
$$

a contradiction. Therefore, $S_{\left\{v_{1}, v_{2}\right\}}=S_{\left\{v_{3}, v_{4}\right\}}=\emptyset$, as required.

Claim 2.2 $S_{\left\{v_{1}, v_{2}, v_{3}\right\}}=S_{\left\{v_{2}, v_{3}, v_{4}\right\}}=\emptyset$.

If $S_{\left\{v_{1}, v_{2}, v_{3}\right\}} \neq \emptyset$, then $G$ contains $H_{2}$ and $H_{4}$ as induced subgraphs. Thus, from Lemma 2.6 (ii) and (iv),

$$
\left\{\begin{array}{l}
(1-\theta)=-\frac{1}{d_{v_{2}}} \\
(1-\theta)=-\frac{d_{v_{3}}+d_{v_{5}}}{d_{v_{3}} d_{v_{2}}}
\end{array}\right.
$$

which yield that $d_{v_{5}}=0$, a contradiction.

Similarly, if $S_{\left\{v_{2}, v_{3}, v_{4}\right\}} \neq \emptyset$, then from Lemma 2.6 (ii) and (iv),

$$
\left\{\begin{array}{l}
(1-\theta)=-\frac{1}{d_{v_{3}}} \\
(1-\theta)=-\frac{d_{v_{3}}+d_{v_{5}}}{d_{v_{3}} d_{v_{2}}}
\end{array}\right.
$$

which yield that $d_{v_{3}}+d_{v_{5}}=d_{v_{2}}$. Notice that any vertex distinct with $v_{3}$ and $v_{5}$ must be 
adjacent to $v_{3}$ or $v_{5}($ thanks to $\nu(G)=2)$. Therefore,

$$
d_{v_{2}}=d_{v_{3}}+d_{v_{5}} \geq 3+3+n-6=n
$$

a contradiction.

Claim $2.3 S_{\left\{v_{1}, v_{3}, v_{4}\right\}}=\emptyset$.

Assume that $S_{\left\{v_{1}, v_{3}, v_{4}\right\}} \neq \emptyset$ and $v_{6} \in S_{\left\{v_{1}, v_{3}, v_{4}\right\}}$. If $v_{5} \sim v_{6}$, then $X_{4}$ (see Fig. 3) is an induced subgraph of $G$. By observation, $X_{4}$ contains an induced subgraph (by deleting $v_{4}$ with incident edges) isomorphic to $H_{6}$ (see Fig. 2), then from Lemma 2.6 (vi)

$$
(1-\theta)=-\frac{1}{d_{v_{5}}}
$$

Combining (17) and the second equation of (15), we obtain that $d_{v_{2}}=0$, a contradiction.

If $v_{5} \nsim v_{6}$, then the principal submatrix, say $M_{6}$, of $\mathcal{L}(G)-\theta I$ indexed by $\left\{v_{1}, \cdots, v_{6}\right\}$ can be written as the following block form

$$
M_{6}=\left(\begin{array}{cc}
M_{4} & \alpha \\
\alpha^{T} & 1-\theta
\end{array}\right),
$$

where $M_{4}$ has been given in the proof of Lemma 2.6 (iv) and

$$
\alpha=\left(\frac{-1}{\sqrt{d_{v_{1}} d_{v_{6}}}}, 0, \frac{-1}{\sqrt{d_{v_{3}} d_{v_{6}}}}, \frac{-1}{\sqrt{d_{v_{4}} d_{v_{6}}}}, 0\right)^{T}
$$

a column vector. Obviously, the Eq. (77) still holds here, and by applying it to the columns of $M_{6}$, we get the equations of (요 and

$$
\frac{-a}{\sqrt{d_{v_{1}} d_{v_{6}}}}-\frac{c}{\sqrt{d_{v_{3}} d_{v_{6}}}}=0
$$

Then from (18) and the values of $a$ and $c$ obtained before

$$
a=-\sqrt{d_{v_{1}} d_{v_{5}}}(1-\theta)\left(\frac{d_{v_{3}}}{d_{v_{5}}}+1\right), \quad c=\sqrt{\frac{d_{v_{3}}}{d_{v_{5}}}},
$$

it follows that $\sqrt{\frac{d_{v_{1}}}{d_{v_{5}}}}=\sqrt{d_{v_{1}} d_{v_{5}}}(1-\theta)\left(\frac{d_{v_{3}}}{d_{v_{5}}}+1\right)$, which yields $(1-\theta)>0$, contradicting with Lemma 2.6 (iv).

Claim $2.4 S_{\left\{v_{1}, v_{2}, v_{3}, v_{4}\right\}}=\emptyset$.

Assume that there is a vertex, say $v_{6}$, in $S_{\left\{v_{1}, v_{2}, v_{3}, v_{4}\right\}}$. If $v_{5} \nsim v_{6}$, then $X_{5}$ (see Fig. 3) is an induced subgraph of $G$. Deleting the vertex $v_{3}$ with the incident edges from $X_{5}$, the resultant graph is isomorphic to $H_{6}$ in Fig. 2. Then from Lemma 2.6 (vi), $(1-\theta)=-\frac{1}{d_{v_{2}}}$, which together with $(1-\theta)=-\frac{d_{v_{3}}+d_{v_{5}}}{d_{v_{3}} d_{v_{2}}}$ (thanks to Lemma 2.6 (iv))indicates that $d_{v_{5}}=0$, a contradiction.

If $v_{5} \sim v_{6}$, then the principal submatrix, say $M_{7}$, of $\mathcal{L}(G)-\theta I$ indexed by $\left\{v_{1}, \cdots, v_{6}\right\}$ 
can be written as

$$
M_{7}=\left(\begin{array}{cc}
M_{4} & \beta \\
\beta^{T} & 1-\theta
\end{array}\right) \text {, }
$$

where $M_{4}$ is as above and

$$
\beta=\left(\frac{-1}{\sqrt{d_{v_{1}} d_{v_{6}}}}, \frac{-1}{\sqrt{d_{v_{2}} d_{v_{6}}}}, \frac{-1}{\sqrt{d_{v_{3}} d_{v_{6}}}}, \frac{-1}{\sqrt{d_{v_{4}} d_{v_{6}}}}, \frac{-1}{\sqrt{d_{v_{5}} d_{v_{6}}}}\right)^{T}
$$

a column vector. Applying (7) to the last column of $M_{7}$, we get

$$
\frac{a}{\sqrt{d_{v_{1}}}}+\frac{b}{\sqrt{d_{v_{2}}}}+\frac{c}{\sqrt{d_{v_{3}}}}=\frac{1}{\sqrt{d_{v_{5}}}}
$$

which together with the second equation of (8) yields that

$$
b\left(\frac{1}{\sqrt{d_{v_{2}}}}+\sqrt{d_{v_{2}}}(1-\theta)\right)=0 .
$$

Since $b \neq 0$ obtained before, then we have $(1-\theta)=-\frac{1}{d_{v_{2}}}$, and thus $d_{v_{5}}=0$ (thanks to $(1-\theta)=-\frac{d_{v_{3}}+d_{v_{5}}}{d_{v_{3}} d_{v_{2}}}$ in Lemma 2.6 (iv)), a contradiction.

In this case, combining Claims 2.1-2.4, we see that all the vertices out of $V\left(P_{4}\right)$ belong to $S_{\left\{v_{1}, v_{2}, v_{4}\right\}}$. Furthermore, it is obvious that $S_{\left\{v_{1}, v_{2}, v_{4}\right\}}$ induces a clique of $G$, as $\nu(G)=2$. Hence, the structure of $G$ is clear now, and $d_{v_{2}}=n-2, d_{v_{3}}=2, d_{v_{4}}=n-3$ and $d_{v_{5}}=n-2$. From Lemma 2.6 (iv),

$$
\begin{aligned}
(1-\theta) & =-\frac{d_{v_{2}}+d_{v_{4}}}{d_{v_{4}} d_{v_{5}}}=\frac{-(2 n-5)}{(n-2)(n-3)} \\
& =-\frac{d_{v_{3}}+d_{v_{5}}}{d_{v_{3}} d_{v_{2}}}=\frac{-n}{2(n-2)},
\end{aligned}
$$

which implies that $n=5$, i.e., $G=H_{4}$. However, $H_{4} \notin \Omega$ by direct calculation. Therefore, $S_{\left\{v_{1}, v_{2}, v_{4}\right\}}=\emptyset$, and by symmetry we get $S_{\left\{v_{1}, v_{3}, v_{4}\right\}}=\emptyset$.

Case 3. Suppose that $v_{5} \in S_{\left\{v_{1}, v_{2}, v_{3}, v_{4}\right\}}$ (i.e., $S_{\left\{v_{1}, v_{2}, v_{3}, v_{4}\right\}} \neq \emptyset$ ) and $S_{\left\{v_{1}, v_{4}\right\}}=S_{\left\{v_{1}, v_{2}, v_{4}\right\}}=$ $S_{\left\{v_{1}, v_{3}, v_{4}\right\}}=\emptyset$.

If this is the case, then the vertices of $V(G) \backslash\left\{V\left(P_{4}\right) \cup S_{\left\{v_{1}, v_{2}, v_{3}, v_{4}\right\}}\right\}$ belong to $S_{\left\{v_{1}, v_{2}\right\}}$, $S_{\left\{v_{3}, v_{4}\right\}}, S_{\left\{v_{1}, v_{2}, v_{3}\right\}}$ or $S_{\left\{v_{2}, v_{3}, v_{4}\right\}}$. Then we have the following claims.

Claim $3.1 S_{\left\{v_{1}, v_{2}\right\}}=S_{\left\{v_{3}, v_{4}\right\}}=\emptyset$.

It suffices to show that $S_{\left\{v_{1}, v_{2}\right\}}=\emptyset$. Suppose $S_{\left\{v_{1}, v_{2}\right\}} \neq \emptyset$ and $v_{6} \in S_{\left\{v_{1}, v_{2}\right\}}$, then

$$
(1-\theta)=-\frac{1}{d_{v_{1}}}
$$

from Lemma 2.6 (i). Since

$$
(1-\theta)=-\frac{d_{v_{2}}+2 d_{v_{4}}}{d_{v_{4}}\left(d_{v_{2}}+d_{v_{5}}\right)}=-\frac{d_{v_{3}}+2 d_{v_{1}}}{d_{v_{1}}\left(d_{v_{3}}+d_{v_{5}}\right)}
$$


from Lemma 2.6 (v), then by (20) and (21) we derive that

$$
\left\{\begin{array}{l}
d_{v_{2}} d_{v_{1}}+2 d_{v_{1}} d_{v_{4}}=d_{v_{2}} d_{v_{4}}+d_{v_{4}} d_{v_{5}} \\
2 d_{v_{1}}=d_{v_{5}}
\end{array}\right.
$$

which implies that $d_{v_{1}}=d_{v_{4}}$. As $G$ contains an induced $P_{4}$, then the equation (1) holds from Lemma 2.5. It follows from (11), (20) and $d_{v_{1}}=d_{v_{4}}$ that

$$
d_{v_{1}}\left(d_{v_{2}}+d_{v_{3}}\right)=d_{v_{2}} d_{v_{3}}
$$

Now we say that $S_{\left\{v_{2}, v_{3}, v_{4}\right\}}=\emptyset$, otherwise $(1-\theta)=-\frac{1}{d_{v_{3}}}$ from Lemma 2.6 (ii), and then $d_{v_{1}}=d_{v_{3}}$ from (20). Thus the equation (22) can be simplified as $d_{v_{3}}=0$, a contradiction. Analogously, one can derive that $S_{\left\{v_{1}, v_{2}, v_{3}\right\}}=\emptyset$. As a result, $d_{v_{2}}=d_{v_{1}}+1$. Recalling that $d_{v_{1}}=d_{v_{4}}$, then $\left|S_{\left\{v_{1}, v_{2}\right\}}\right|=\left|S_{\left\{v_{3}, v_{4}\right\}}\right|$, and thus $d_{v_{2}}=d_{v_{3}}$. Considering (22) again, one can obtain that $d_{v_{1}}=1$, a contradiction.

Claim $3.2 S_{\left\{v_{1}, v_{2}, v_{3}\right\}}=S_{\left\{v_{2}, v_{3}, v_{4}\right\}}=\emptyset$.

It suffices to show that $S_{\left\{v_{1}, v_{2}, v_{3}\right\}}=\emptyset$. Suppose on the contrary that $S_{\left\{v_{1}, v_{2}, v_{3}\right\}} \neq \emptyset$, then $d_{v_{2}}=d_{v_{3}}$ by observation. From Lemma 2.6 (ii), we have $1-\theta=-\frac{1}{d_{v_{2}}}$. Thus the equation (11) of Lemma 2.5 can be simplified as

$$
d_{v_{1}}+d_{v_{4}}=d_{v_{2}}
$$

It is not hard to see that

$$
\left\{\begin{array}{l}
d_{v_{1}}=\left|S_{\left\{v_{1}, v_{2}, v_{3}\right\}}\right|+\left|S_{\left\{v_{1}, v_{2}, v_{3}, v_{4}\right\}}\right|+1 \\
d_{v_{4}}=\left|S_{\left\{v_{2}, v_{3}, v_{4}\right\}}\right|+\left|S_{\left\{v_{1}, v_{2}, v_{3}, v_{4}\right\}}\right|+1 \\
d_{v_{2}}=\left|S_{\left\{v_{1}, v_{2}, v_{3}\right\}}\right|+\left|S_{\left\{v_{2}, v_{3}, v_{4}\right\}}\right|+\left|S_{\left\{v_{1}, v_{2}, v_{3}, v_{4}\right\}}\right|+2
\end{array}\right.
$$

which together with (23) implies that $\left|S_{\left\{v_{1}, v_{2}, v_{3}, v_{4}\right\}}\right|=0$, a contradiction. Therefore, the results of Claim 3.2 hold.

Now we are in a position to complete Case 3. Combining Claims 3.1 and 3.2, we see that all vertices out of $V\left(P_{4}\right)$ belong to $S_{\left\{v_{1}, v_{2}, v_{3}, v_{4}\right\}}$. Then $d_{v_{2}}=d_{v_{1}}+1$. We claim that $S_{\left\{v_{1}, v_{2}, v_{3}, v_{4}\right\}}$ induces a clique of $G$. Otherwise, there exist two vertices, say $v_{5}$ and $v_{6}$, of $S_{\left\{v_{1}, v_{2}, v_{3}, v_{4}\right\}}$, which are not adjacent. Then the subgraph induced by $\left\{v_{1}, v_{2}, v_{4}, v_{5}, v_{6}\right\}$ is isomorphic to $H_{6}$ in Fig. 2. Hence by Lemma 2.6 (vi), $1-\theta=-\frac{1}{d_{v_{1}}}=-\frac{1}{d_{v_{2}}}$, which indicates that $d_{v_{2}}=d_{v_{1}}$, contradicting with $d_{v_{2}}=d_{v_{1}}+1$. As a result, if $\left|S_{\left\{v_{1}, v_{2}, v_{3}, v_{4}\right\}}\right| \geq 3$, then $1+\frac{1}{n-1}$ is an $\mathcal{L}$ eigenvalue of $G$ with multiplicity at least 2 . Since $G \neq K_{n}$, then $\rho_{n-1}(G) \leq 1$ by Lemma 2.2. Clearly, $G \varsubsetneqq K_{p, q}, K_{a} \vee(n-a) K_{1}$, then $\rho_{2}(G) \geq \frac{n-1}{n-2}$ by Lemma 2.1. Noting that $\rho_{n}(G)=0$, we obtain $G \notin \Omega$. For the case of $\left|S_{\left\{v_{1}, v_{2}, v_{3}, v_{4}\right\}}\right| \leq 2$, one can get $G \notin \Omega$ by direct calculation.

The necessity part can be proved by Cases 1-3, and then the proof is completed.

\section{Acknowledgements}

The authors thank the anonymous referees for their valuable comments of this paper. This 
work is supported by the Natural Science Foundation of Shandong Province (No. ZR2019BA016).

\section{References}

[1] E.R. van Dam, G.R. Omidi, Graphs whose normalized Laplacian has three eigenvalues, Linear Algebra Appl. 435 (2011) 2560-2569.

[2] J. Li, J.M. Guo, W.C. Shiu, Bounds on normalized Laplacian eigenvalues of graphs, J. Inequal. Appl. 316 (2014) 1-8.

[3] R.O. Braga, R.R. Del-Vecchio, V.M. Rodrigues, V. Trevisan, Trees with 4 or 5 distinct normalized Laplacian eigenvalues, Linear Algebra Appl. 471 (2015) 615-635.

[4] J. Guo, J. Li, W.C. Shiu, The largest normalized Laplacian spectral radius of non-bipartite graphs, Bull. Malaysian Math. Sci. Soc. 39 (1) (2016) 77-87.

[5] K.C. Das, S. Sun, Extremal graph on normalized Laplacian spectral radius and energy, Elect. J. Linear Algebra, 29 (2016) 237-253.

[6] X. Huang, Q. Huang, On graphs with three or four distinct normalized Laplacian eigenvalues, Algebra Colloquium, 26:1 (2019) 65-82.

[7] S. Sun, K.C. Das, On the second largest normalized Laplacian eigenvalue of graphs, Appl. Math. Comput. 348 (2019) 531-541.

[8] S. Sun, K.C. Das, Normalized Laplacian spectrum of complete multipartite graphs, Discrete Appl. Math. 284 (2020) 234-245.

[9] S. Sun, K.C. Das, On the multiplicities of normalized Laplacian eigenvalues of graphs, Linear Algebra Appl. 609 (2021) 365-385.

[10] F.R. Chung, Spectral Graph Theory, American Mathematical Society, Providence, RI, 1997.

[11] F. Tian, D. Wong, S. Wang, Characterization of graphs with some normalized Laplacian eigenvalue of multiplicity $n-3$, Linear Algebra Appl. 606 (2020) 127-143.

[12] F. Tian, J. Cai, Z. Liang, X. Su, On graphs with some normalized Laplacian eigenvalue of extremal multiplicity, arXiv:2007.11844v1.

[13] A.E. Brouwer, W.H. Haemers, Spectra of Graphs, Springer, New York, 2012. 